\title{
TUBERCULAR CHOROIDITIS MASQUERADING AS CENTRAL SEROUS CHORIORETINOPATHY - A CASE REPORT
}

Sandhya Ramachandra ${ }^{1}$, Sameera Veerakanellore ${ }^{2}$

\section{HOW TO CITE THIS ARTICLE:}

Sandhya Ramachandra, Sameera Veerakanellore. "Tubercular choroiditis masquerading as central serous chorioretinopathy - a case report". Journal of Evolution of Medical and Dental Sciences 2013; Vol. 2, Issue 45, November 11; Page: 8760-8764.

ABSTRACT: Tuberculosis can involve essentially any ocular tissue. Tubercular Uveitis is difficult to diagnose because it may occur in patients without systemic manifestations of Tuberculosis. The diagnosis is therefore often presumptive, based on indirect evidence such as a positive skin test and negative findings for other causes of uveitis since a definitive diagnosis would require identification of the causative organism itself which is difficult to obtain. While the diagnosis of Central Serous Chorioretinopathy(CSCR) can be straight forward and accurate in majority of the cases, some unusual cases suchas this require closer monitoring. A diagnosis of Tuberculosis should be in the backdrop of even typical appearing CSCR in India. A scar in the fundus of the other eye and a vague history of lymphadenopathy in childhood were the only suspicious factors in this case. Following investigations, a definitive diagnosis of choroidaltuberculosis was established, therapy instituted and resolution observed. The visual acuity recovered significantly after ATT and steroids.

KEY-WORDS: CSCR, OCT ,Tubercular choroiditis

INTRODUCTION: The two species causing TB in humans are the human strain M. tuberculosis, which is acquired by inhaling infected airborne droplets, and the bovine strain M. bovis, which is acquired by drinking unpasteurized milk from infected cattle. ${ }^{1}$ Ocular Tuberculosis can present with a myriad of symptoms and signs. Here we present a case report of TubercularChoroiditis that presented atypically in a young, male patient.

CASE SUMMARY: A 32 year oldmale presented to eye department of PES medical college,Kuppam, rural Andhra Pradesh,in the month of June 2013 withcomplaints of sudden diminution of vision in left eye since that morning. He also gave history of seeing distorted images in the same eye. No history of trauma/redness/pain. He is a known hypertensive since two years, not on regular medication.

On questioning repeatedly history of hospitalization in childhood for bilateral inguinal swellings, the details of which were unavailable, was elicited.

Examination revealed normal anterior segments and pupillary reactionsin both eyes. Vision was 6/6 inright eye(RE) and 6/60 improving to 6/18 with pinhole in left eye(LE). REfundoscopy showed a well circumscribed circular scar of $250 \mu$ in the area inferonasal to the macula(Fig.1). In the LE, an elevated circular lesion with ring reflex was seen in the macula with absent foveal reflex. Initial provisional diagnosis of LE - CSCR was made.

On general physical examination, patient's blood pressure was found to be $160 / 120 \mathrm{mmHg}$. Patient was referred to physician for immediate systemic management of high BP. 
Patient was evaluated two days later, for ophthalmic investigations. Incidentally, deterioration of vision to counting fingers at $1 \mathrm{~m}$ was noted along with increase in size of the lesion with hemorrhagic spotsin LE(Fig.2)

INVESTIGATIONS: Ophthalmic investigations OCT revealed serous retinal detachment in left eye(Fig.3). FFA revealedhyper-fluorescence, increasing in size and intensity in late phasein the supero-temporal Macula(Fig.4).B-scan showed thickening of choroid(Fig.5), in thecorresponding zone, with altered echoes.

Chest x-ray, Mantouxtest,serology for Toxoplasma and HIV were done along with other routine laboratory investigations- Hb, TC, DC, ESR. Mantoux test read 28x30mm after 48hrs(Fig.6). Chest Xray was normal(Fig.7).Other investigations were with in normal range.

TREATMENT: A diagnosis of Tubercular Choroiditis was made and patient started on Category-1 Anti Tubercular Therapy(ATT), under the care of chest physician. Patient was reviewed every $3^{\text {rd }}$ day. Systemic steroids were started 10 days after ATT.

FOLLOW UP: Improvement in vision in LE to 6/60 along with decrease in size of lesion with disappearance of hemorrhagic spots notedby $12^{\text {th }}$ day of ATT(Fig.8).Two weeks later, with adjunctive oral corticosteroids,vision improved to $6 / 9$ in the affected eye with fundus showing choroidal tubercle like picture (Fig. 9) After 4weeks of initiating treatment, vision improved to 6/6.

DISCUSSION: Tuberculosis is known to cause a myriad of ophthalmic pathology.Approximately, 2 billion people are affected worldwide with tuberculosis, while only $10 \%$ of these people have clinical manifestations. ${ }^{2}$ The absence of pulmonary tuberculosis does not rule out ocular tuberculosis. ${ }^{3}$ Primary infection in the eye typically presents as corneal,conjunctival and scleral disease which is rare. Secondary ocular infection results from hematogenous spread of the organism or from a hypersensitivity reaction to extraocular infection. ${ }^{4}$

The clinical ocular manifestation of secondary infection is highly variable and depends on several factors, including the virulence of the organism, the degree of tissue hypersensitivity to the infection, and the extent of acquired host resistance. ${ }^{4}$ Choroidal tubercles are the most common manifestation of intraocular tuberculosis.5,6,7It may also present as multifocal progressive or diffuse choroiditis resembling serpiginous choroiditis. ${ }^{8}$

Various criteria have been periodically updated for the diagnosis of the same. Though definitive diagnosis of ocular tuberculosis is established by the demonstration of the mycobacterium with the help of culture or DNA amplification from ocular samples, a presumptive diagnosis can be made based on ancillary testing after other causes of uveitis have been ruled out. AMantoux test can be done if ocular tuberculosis is suspected. ${ }^{2,3}$ Greater than $5 \mathrm{~mm}$ of induration after $48-72$ hours is a positive result in a HIV patient. 2,9 Greater than $10 \mathrm{~mm}$ of induration is considered positive result in high-risk individuals, such as in people living in endemic areas, health-care workers and nursing home patients. ${ }^{2,9}$ Induration greater than $15 \mathrm{~mm}$ is considered a positive result in all cases. ${ }^{2,9}$

The present case had no signs of anterior segment or posterior segment inflammation which could have led to investigations for tuberculosis. A typical CSCR like picture with the backdrop of 
systemic hypertension, was almost definitive. However, a small scar in the Right eye and vague history of ?lymph node biopsy in childhood led to further investigations to rule out tuberculosis.

CONCLUSION: Ocular Tuberculosis can have variable clinical manifestations. Patient's gender, age, his active life style and history of hypertension, initial clinical presentation and fundus findings led us to think in terms of CSCR. A high index of clinical suspicion is necessary especially in a country like ours where the disease is still rampant.

\section{REFERENCES:}

1. Kanski, Jack J. Clinical Ophthalmology: A systemic approach.- $7^{\text {thedition. Elsevier }}$ Saunders;2011. Chapter 11;p.450.

2. Gupta V, Gupta A, Rao NA. Intraocular tuberculosis - an update. SurvOphthalmol 2007;52(6):561-87.

3. Bodaghi B, Le Hoang P. Ocular tuberculosis. CurrOpinOphthalmol 2000;11(6):443-8.

4. Albert \&Jacobiec's- Principles and Practice of Ophthalmology.- 3rd edition. Elsevier Inc;2008. Chapter 95

5. Massaro D, Katz S, Sachs M. Choroidal tubercles. A clue to hematogenous tuberculosis. Ann Inter Med. 1964;60:231-41.

6. Grewal A, Kim RY, Cunningham ET Jr. Miliary tuberculosis. Arch Ophthalmol. 1998;116:9534.

7. Gupta V, Gupta A, Sachdeva N, et al. Simultaneous choroidaltuberculoma and epididymoorchitis caused by Mycobacterium tuberculosis. Am J Ophthalmol.2005;140:310-12.

8. Vishali Gupta, Amod Gupta, Sunil Arora, PradeepBambery, Mangat Ram Dogra, Anita Agarwal, Presumed tubercular serpiginouslikechoroiditis: Clinical presentations and management, Ophthalmology, Volume 110, Issue 9, September 2003, Pages 1744-1749.

9. Gupta A, Gupta V. Tubercular posterior uveitis. IntOphthalmolClin 2005;45(2):71-88.

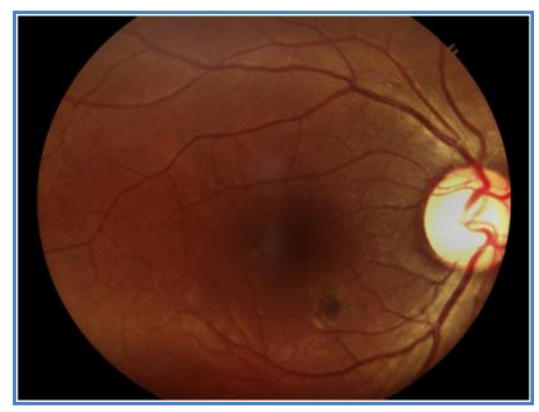

(Fig.1) Right Eye fundus picture of the patient

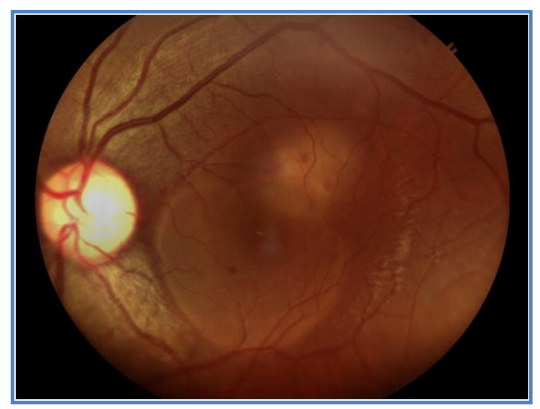

(Fig.2) LE fundus picture of the patient on second visit.

The size of lesion has increased significantly with appearance of hemorrhagic spots. 


\section{CASE REPORT}

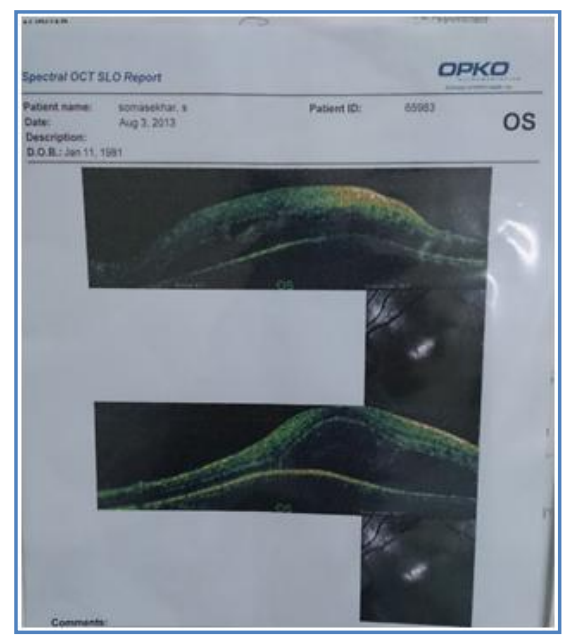

(Fig.3) OCT of LE shows Retinal Detachment
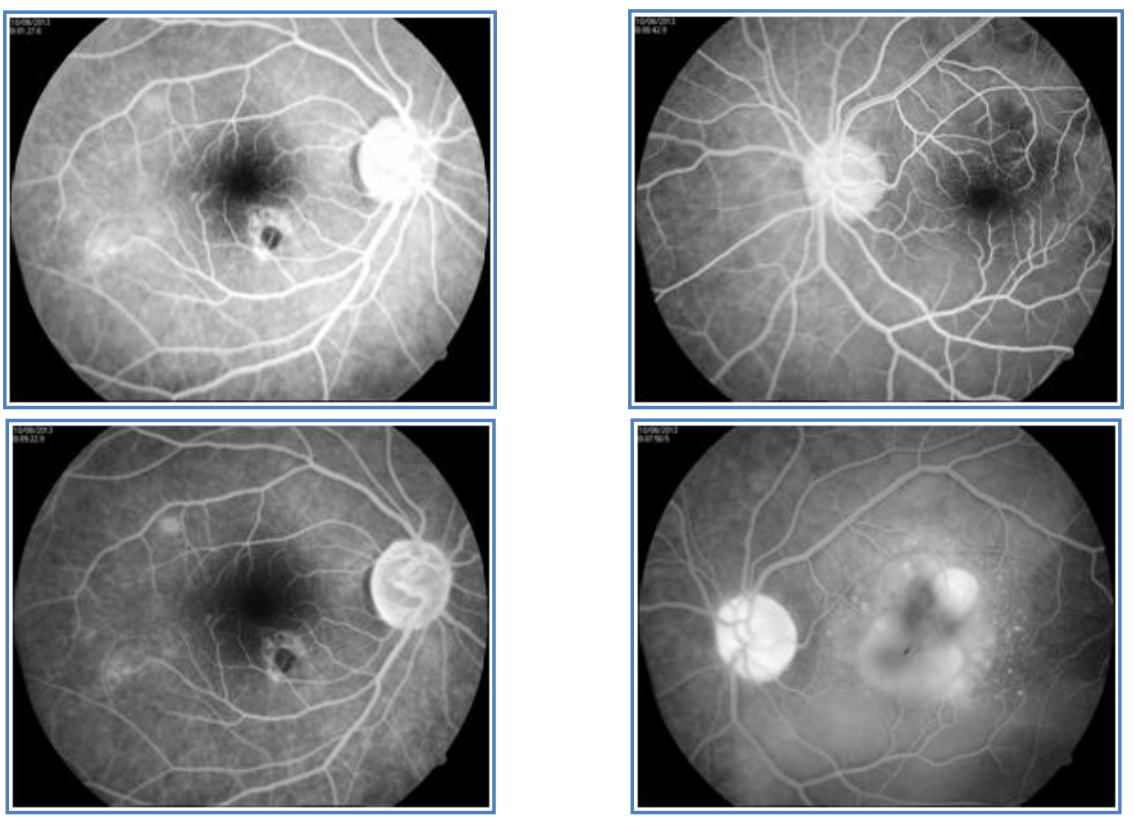

(Fig.4) FFA shows blocked fluorescence inferotemporal to the disc in RE. LE shows late hyperflourescence.

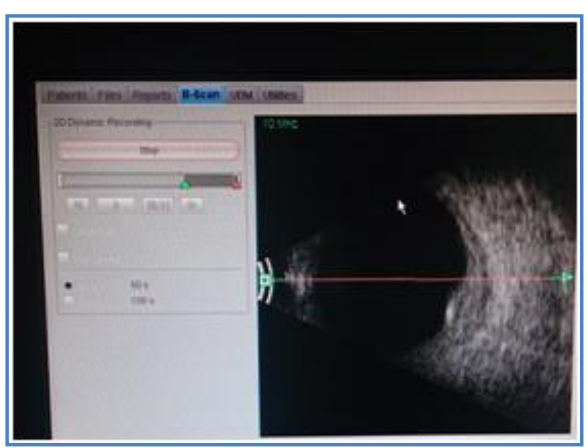

(Fig.5) B-Scan shows choroidal thickening.

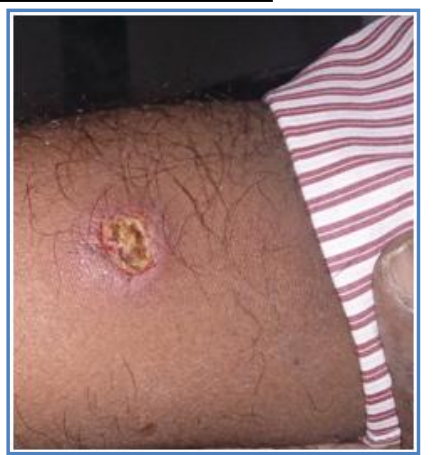

(Fig.6) Mantoux test after 48hours showing significant induration. 


\section{CASE REPORT}

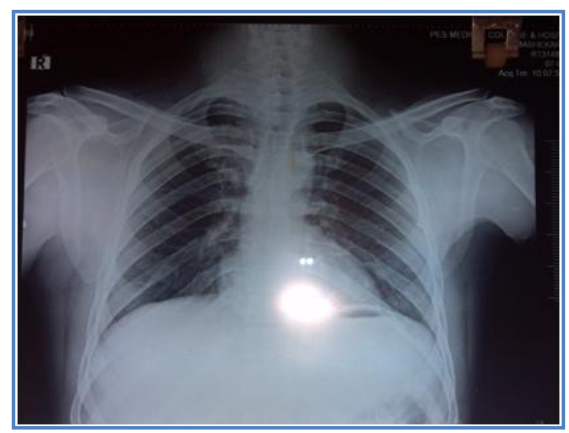

(Fig.7) Chest X-ray PA view.

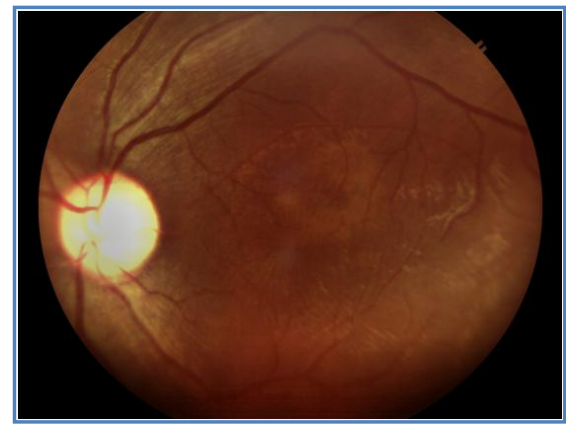

(Fig. 8) Fundus picture of LE after

starting ATT. VA was 6/60

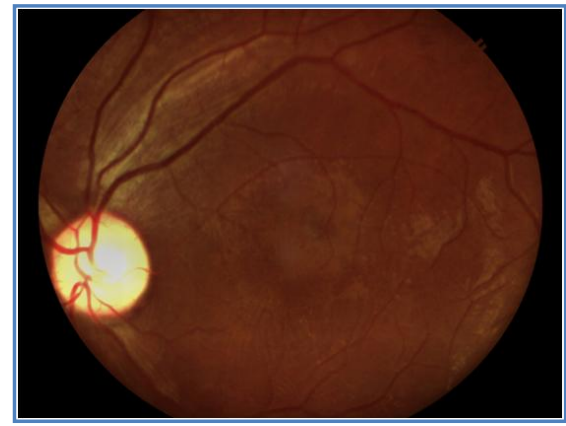

(Fig. 9) Fundus picture at subsequent follow up. VA improved to $6 / 9$.

\section{AUTHORS:}

1. Sandhya Ramachandra

2. Sameera Veerakanellore

\section{PARTICULARS OF CONTRIBUTORS:}

1. Professor, Department of Ophthalmology, PES Institute of Medical Sciences \& Research, Kuppam, Andhra Pradesh.

2. PG Resident, Department of Ophthalmology, PES Institute of Medical Sciences \& Research, Kuppam, Andhra Pradesh.

\section{NAME ADDRESS EMAIL ID OF THE} CORRESPONDING AUTHOR:

Dr. Sameera Veerakanellore,

PG Resident,

PESIMSR, Kuppam, Andhra Pradesh - 517425.

Email - sameeranikhil@gmail.com

Date of Submission: 21/10/2013.

Date of Peer Review: 22/10/2013.

Date of Acceptance: 28/10/2013.

Date of Publishing: 06/11/2013 